\title{
On Bridging The Gap Between Homogeneous and Heterogeneous Rendezvous Schemes for Cognitive Radios
}

\author{
Ching-Chan Wu, Shan-Hung Wu \\ Dept. of Computer Science, National Tsing Hua University \\ Hsinchu, Taiwan, ROC \\ ccwu@ netdb.cs.nthu.edu.tw, shwu@cs.nthu.edu.tw
}

\begin{abstract}
Cognitive radio allows radio devices to access the idle spectrum opportunistically, thus alleviates the huge demand for spectrum. Rendezvous, where two radios complete handshaking in an idle channel, is a key step for cognitive radios to start communication. Radios may have the same (homogeneous) or different (heterogeneous) spectrum sensing capabilities. Currently, there is a "gap" between the rendezvous algorithms for homogeneous and heterogeneous cognitive radios - existing homogeneous algorithms incur high delay when applied to heterogeneous radios; while heterogeneous algorithms incur high congestion when applied to homogeneous radios. Since mixtures of these two types of radios appear commonly in practice, it is crucial to bridge the gap between the respective rendezvous algorithms. In this paper, we propose a new rendezvous algorithm, named the ICH scheme, for arbitrary mixtures of radios with homogeneous or heterogeneous spectrum sensing capabilities. Rigorous analysis and extensive simulations are conducted and show that ICH is the first rendezvous scheme that guarantees rendezvous for arbitrary mixtures of homogeneous and heterogeneous radios without incurring large delay and congestion.
\end{abstract}

\section{Categories and Subject Descriptors}

C.2.1 [Network Architecture and Design]: Wireless communication; C.2.4 [Distributed Systems]: Distributed applications

\section{Keywords}

cognitive radio; blind rendezvous; homogeneous; heterogeneous

\section{INTRODUCTION}

The tremendous demand for the radio spectrum continues growing, as more and more wireless devices have spread around people in the past few years. Due to the fixed and

Permission to make digital or hard copies of all or part of this work for personal or classroom use is granted without fee provided that copies are not made or distributed for profit or commercial advantage and that copies bear this notice and the full citation on the first page. Copyrights for components of this work owned by others than ACM must be honored. Abstracting with credit is permitted. To copy otherwise, or republish, to post on servers or to redistribute to lists, requires prior specific permission and/or a fee. Request permissions from permissions@acm.org.

MobiHoc'13, July 29-August 1, 2013, Bangalore, India.

Copyright 2013 ACM 978-1-4503-2193-8/13/07 ...\$15.00. uneven spectrum allocation, the spectrum resources are inadequate for applications in some places. However, the spectrum is rarely used cross channels, time, and space continuously $[11,1]$. There are usually spectrum holes, which consist of idle channels, at some time and space. To alleviate the spectrum demand, cognitive radio is proposed as a means of DSA [1] to utilize those spectrum holes by allowing the Secondary Users (SUs, or simply radios) to sense the spectrum ranging within their own device capability and to dynamically tune into different idle channels not currently used by Primary Users (PUs) to communicate with each other opportunistically.

Rendezvous is a key step for cognitive radios to start communication. Two radios are said to rendezvous with each other if they complete handshaking (for the purposes of neighbor discovery, data transmission, etc.) in an idle channel. One popular technique to guide a pair of radios to rendezvous is to use the Channel Hopping $(\mathrm{CH})$ schemes [5, $9,10,13,14,17,18]$. A CH scheme, programmed in each radio in a network, divides the time of a radio evenly into time slots, and requires the radio to hop to a sequence of channels in some predefined order at consecutive slots. This sequence is called the $\mathrm{CH}$ sequence for that radio. The $\mathrm{CH}$ scheme ensures that, by following their $\mathrm{CH}$ sequences, two radios can rendezvous with each other within a finite delay, called Time To Rendezvous (TTR). In contrast to other centralized techniques $[6,7], \mathrm{CH}$ schemes allow radios to obtain their own $\mathrm{CH}$ sequences in a distributed manner, thereby walking around a single point of failure. $\mathrm{CH}$ schemes also help avoid congestion, since at each time slot, different radios may hop to different channels. Recent $\mathrm{CH}$ schemes $[14,21,4,16]$ give another advantage that rendezvous can be guaranteed without assuming timer synchronization between radios. Since timer synchronization is hard to achieve in practice (especially before rendezvous), these schemes have broader applicability. In this paper, we focus on $\mathrm{CH}$ schemes for asynchronous radios.

Existing $\mathrm{CH}$ schemes focus on either homogeneous or heterogeneous cognitive radios. Let $V_{i}$ be the spectrum sensing capability of a radio $i$ (that is, a set of channels with which the radio $i$ is capable of sensing), and $P_{i}$ be a set channels in $V_{i}$ that are detected to be occupied by PUs. Homogeneous $\mathrm{CH}$ schemes assume radios to have homogeneous sensing capability, i.e., $V_{i}=V_{j}=V$, and guarantees rendezvous if $\left(V \backslash P_{i}\right) \cap\left(V \backslash P_{j}\right) \neq \emptyset$ within a worst-case delay, called Maximum Time To Rendezvous (MTTR), of $O\left(|V|^{2}\right)$ slots [14, 21, 4]. Heterogeneous $\mathrm{CH}$ schemes, on the other hand, as- 
sume $V_{i} \neq V_{j}$ and guarantees rendezvous within $O\left(\left|V_{i}\right|\left|V_{j}\right|\right)$ MTTR [18, 19] if $\left(V_{i} \backslash P_{i}\right) \cap\left(V_{j} \backslash P_{j}\right) \neq \emptyset$.

We observe a "gap" between the homogeneous and heterogeneous $\mathrm{CH}$ schemes. Applying homogeneous schemes to heterogeneous radios results in either loss of the guarantee (if we let the $\mathrm{CH}$ scheme generate a $\mathrm{CH}$ sequence for radio $i$ using $V_{i}$ directly) or $O\left(|U|^{2}\right) \operatorname{MTTR}$ (if we let $V_{i}=U$ for all $i$ and regard channels in $U \backslash V_{i}$ as occupied, where $U,|U| \gg\left|V_{i}\right|$, is the set of universal channels) which is too high to make the schemes feasible. Similarly, applying heterogeneous schemes to homogeneous radios either loses the rendezvous guarantee [18] or incurs serious congestion [19]. In real networks, mixtures of homogeneous and heterogeneous radios are common. For example, there may be radios from different troop/organizations in a network, and radios from the same troops/organization are likely to have the same spectrum sensing capabilities. It is crucial to have a new rendezvous technique that bridges the gap between homogeneous and heterogeneous $\mathrm{CH}$ schemes.

In this paper, we propose a new $\mathrm{CH}$ scheme, named the Interlocking Channel Hopping ( $\mathrm{ICH}$ ) scheme that guarantees rendezvous for arbitrary mixtures of homogeneous and heterogeneous radios. In addition, the $\mathrm{ICH}$ scheme is carefully designed to achieve two goals - minimizing the MTTR for heterogeneous radios and minimizing the level of congestion (called load, to be explained later) for homogeneous radios - that are currently conflicting due to the aforementioned gap.

To the best of our knowledge, the ICH scheme is the first $\mathrm{CH}$ scheme that guarantees rendezvous for both homogeneous and heterogeneous radios without incurring large delay and congestion. This study largely increases the practicability of $\mathrm{CH}$ schemes to real networks. Following summarizes our contributions:

- We identify a gap between the homogeneous and heterogeneous $\mathrm{CH}$ schemes and propose the ICH scheme that guarantees rendezvous between radios $i$ and $j$ as long as $\left(V_{i} \backslash P_{i}\right) \cap\left(V_{j} \backslash P_{j}\right) \neq \emptyset$, no matter $V_{i}=V_{j}$ or $V_{i} \neq V_{j}$.

- The ICH scheme ensures $O\left(|V|^{2}\right)$ MTTR when $V_{i}=$ $V_{j}=V$, which is the same as the shortest MTTR achieved by existing homogeneous schemes [14, 21, 4]. When $V_{i} \neq V_{j}$, the ICH scheme ensures $O\left(\left|V_{i}\right|\left|V_{j}\right|\right)$ MTTR, which is again as short as the best MTTR achieved by current heterogeneous scheme [18, 19].

- We study the degrees of congestion (denoted by load) for cognitive radios, and carefully design our scheme without incurring congestion. The simulation results show that the load of ICH is very close to the optimal load, $E[l o a d]_{o p t}=\frac{1}{|D|}$, as $\left|V_{i}\right|$ is usually not small.

- The ICH scheme takes into account the clock shift between radios, therefore supports both synchronous and asynchronous environments.

- Extensive simulations are conducted and the results show that under various combination of radios, our scheme is either 10 times faster than extensions of existing homogeneous $\mathrm{CH}$ schemes, or incurs $50 \%$ lighter load than existing heterogeneous $\mathrm{CH}$ schemes.

The rest of this paper is organized as follows. In section 2, we formally define the problem and review a $\mathrm{CH}$ scheme

\begin{tabular}{c|l} 
Variable & \multicolumn{1}{|c}{ Description } \\
\hline$c_{x}$ & The channel numbered $x$ \\
$U$ & The set of universal channels \\
$V_{i}$ & Device capability of radio $i$ \\
$P_{i}$ & The set of PU occupied channels that radio $i$ \\
& detects \\
$s t a r t_{i}$ & The starting channel of $V_{i}$ \\
$t_{i}^{[x]}$ & The $x^{t h}$ time slot of radio $i$ \\
$S_{i}^{[x]}$ & CH sequence of radio $i$ in the $x^{t h}$ round \\
$s_{i}^{[x, 0]}$ & The $y^{t h}$ element in $S_{i}^{[x]}$ the $x^{t h}$ round \\
$F_{i}$ & The fixed subsequence of $S_{i}$ \\
$R_{i}$ & The rotating subsequence of $S_{i}$ \\
$N_{i}$ & The insurance subsequence of $S_{i}$ \\
$k_{i}$ & Rotating amount of $R_{i}$ \\
$M_{i}$ & The rotating subsequence of $N_{i}$ \\
$B_{i}$ & The insurance subsequence of $N_{i}$ \\
$a_{i}$ & Rotating amount of $M_{i}$ \\
$b_{i}$ & The insurance channel of $B_{i}$ \\
$D$ & The set of all radios.
\end{tabular}

Table 1: Notation.

that is relevant to our study. We then explain why minimizing the MTTR for heterogeneous radios and minimizing the load for homogeneous radios are conflicting goals in existing $\mathrm{CH}$ schemes, and propose the ICH scheme for these two goals in Section 3. Section 4 evaluates the performance of our proposals. In Section 5, we review existing works on rendezvous for cognitive radios. Finally, Section 6 concludes the paper.

\section{PRELIMINARIES}

In this section, we formally define the rendezvous problem. We also review state-of-the-art CH schemes. Table 1 lists the notations used throughout this paper.

\subsection{Problem Definition}

Assume that the universal spectrum can be divided into a set $U=\left\{c_{0}, c_{1}, \cdots, c_{|U|-1}\right\}$ of channels. Each radio $i$ can sense a range of spectrum consisting of a set $V_{i}=$ $\left\{c_{x}, c_{x+1}, \cdots, c_{x+\left|V_{i}\right|-1}\right\}$ of continuous channels starting from $c_{x}[12,2,8,11,1]$. We denote $c_{x}$ as start $_{i}$. Each channel in $V_{i}$ is either occupied by nearby Primary Users (PUs) or available for opportunistic usage, and we let $P_{i}$ be the set of PU occupied channels that radio $i$ detects. Two radios $i$ and $j$ are said to have capability-overlap if they can sense common channels, i.e., $V_{i} \cap V_{j} \neq \emptyset$. The time of each radio $i$ is divided evenly into time slots, denoted as $t_{i}^{[0]}, t_{i}^{[1]}, \cdots$. We do not assume any timer synchronization between radios. So given an index $x$, slots $t_{i}^{[x]}$ and $t_{j}^{[x]}$ of two radios $i$ and $j$ may have arbitrary shift in time. We say that two slots $t_{i}^{[x]}$ and $t_{j}^{[y]}$ have time-overlap if they overlap for an interval longer than half of a slot, as shown in Fig. 1.

We adopt the channel hopping scheme, where each radio hops to a channel at each time slot and waits for rendezvous with other radios. Specifically, given a Channel Hopping (CH) sequence $S_{i}=\left[s_{i}^{[0]}, s_{i}^{[1]}, \cdots\right]$, where $s_{i}^{[x]} \in V_{i}$, the radio $i$ hops to channel $s_{i}^{[0]}$ at slot $t_{i}^{[0]}$, and $s_{i}^{[1]}$ at slot $t_{i}^{[1]}$, and so on.

Definition 2.1 (Rendezvous). Given a pair of capabilityoverlapping radios $i$ and $j$ in a network, the radios $i$ and $j$ 


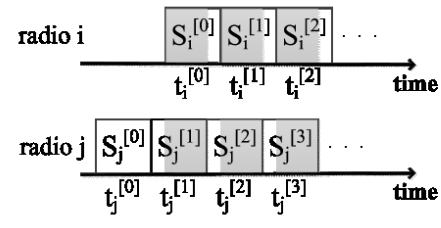

Figure 1: Despite of the asynchronous timers, a slot of radio $i$ must overlap with one slot of radio $j$ over an interval (shaded) longer than half of a slot. For example, the slot $t_{i}^{[1]}$ is time-overlapping with $t_{j}^{[2]}$, but not with $t_{j}^{[3]}$.

rendezvous if $s_{i}^{[x]}=s_{j}^{[y]}=c$ for some $x, y$, and $c$, where $t_{i}^{[x]}$ time-overlaps with $t_{j}^{[y]}$ and $c$ is in both $V_{i} \backslash P_{i}$ and $V_{j} \backslash P_{j}$.

Two radios are said to rendezvous if they hop to some common available channel at a pair of time-overlapping slots. We assume that the duration of a time slot is set long enough such that the handshaking (for, say, neighbor discovery or data transmission) can be done within half of a slot at which rendezvous takes place $[5,18]$.

We formally define our problem as follows:

Problem 2.2. Design a $\mathrm{CH}$ scheme such that a) given any pair of capability-overlapping radios $i$ and $j$ in a network, the scheme is able to return two CH sequences $S_{i}$ and $S_{j}$ and guarantee that by following $S_{i}$ and $S_{j}$ respectively, the radios $i$ and $j$ will rendezvous within finite delay (called Time to Rendezvous) as long as $\left(V_{i} \backslash P_{i}\right) \cap\left(V_{j} \backslash P_{j}\right) \neq \emptyset$; and b) at any time slot, the number of radios which rendezvous on a particular channel should be minimized to avoid congestion.

Note that it is impossible for two radios to rendezvous if they have no available channels in common, e.g., $V_{i} \cap V_{j}=\emptyset$ or $\left(V_{i} \backslash P_{i}\right) \cap\left(V_{j} \backslash P_{j}\right)=\emptyset$.

To simplify the delay analysis, one common metric is the Maximum Time to Rendezvous (MTTR), which measures the maximum time (in number of slots) required for two radios to rendezvous. The shorter the MTTR the better.

\subsection{State of the Arts}

Many $\mathrm{CH}$ schemes are proposed for the rendezvous problem, and can be generally classified into the homogeneous schemes $[14,21,4]$ (which assume $V_{i}=V_{j}$ for all radios $i$ and $j$ ) and heterogeneous $[16,18,19]$ schemes $\left(V_{i} \neq V_{j}\right)$. Next, we briefly summarize the HH scheme [19] as it provides some lemmas that are useful to our study.

To start, we need to extend the notation for a $\mathrm{CH}$ sequence first. A CH sequence $S_{i}=\left[s_{i}^{[0]}, s_{i}^{[1]}, \cdots\right]$ can be partitioned evenly into rounds $S_{i}^{[x]}=\left[s_{i}^{[x, 0]}, s_{i}^{[x, 1]}, \cdots, s_{i}^{\left[x,\left|S_{i}^{[x]}\right|\right]}\right]$, where $s_{i}^{[x, y]}$ denotes the $y^{\text {th }}$ element in the $x^{\text {th }}$ round, as shown in Fig. 2. Note that $s_{i}^{[x, y]}=s_{i}^{\left[x \cdot\left|S_{i}^{[x]}\right|+y\right]}$.

In the $\mathrm{HH}$ scheme, we partition $S_{i}$ into rounds of length 3:

$$
s_{i}^{[x, y]}= \begin{cases}f_{i}^{[x]}, & y=0 \\ r_{i}^{[x]}, & y=1, \\ n_{i}^{[x]}, & y=2,\end{cases}
$$

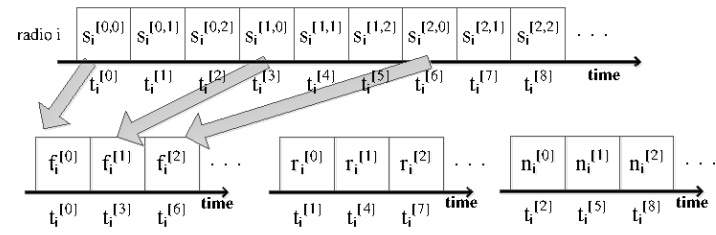

Figure 2: An example $\mathrm{CH}$ sequence. $S_{i}$ is divide into the fixed $F_{i}$, rotating $R_{i}$, and insurance $N_{i}$ subsequences.

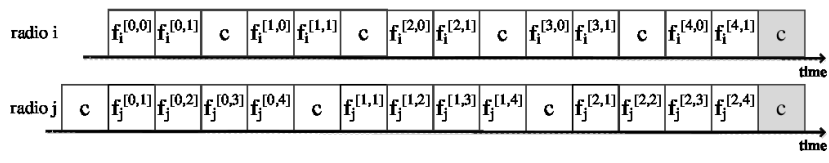

Figure 3: Rounded fixed sequences $F_{i}$ and $F_{j}$ with a common available channel $c$, where $\left|F_{i}^{[x]}\right|=3,\left|F_{j}^{[x]}\right|=$ 5. The MTTR is bounded by $O\left(\left|F_{i}^{[x]}\right|\left|F_{j}^{[x]}\right|\right)$ time slots.

and denote the three elements in each round $x, f_{i}^{[x]}, r_{i}^{[x]}$, $n_{i}^{[x]}$, respectively. This effectively divide $S_{i}$ into three subsequences, namely the fixed sequence $F_{i}=\left[f_{i}^{[0]}, f_{i}^{[1]}, \cdots\right]$, rotating sequence $R_{i}=\left[r_{i}^{[0]}, r_{i}^{[1]}, \cdots\right]$, and insurance sequence $N_{i}=\left[n_{i}^{[0]}, n_{i}^{[1]}, \cdots\right]$ (see Fig. 2).

The fixed sequence $F_{i}$ is further partitioned into rounds $F_{i}^{[x]}=\left[f_{i}^{[x, 0]}, f_{i}^{[x, 1]}, \cdots, f_{i}^{\left[x,\left|F_{i}^{[x]}\right|-1\right]}\right]$. Let $\left|F_{i}^{[x]}\right|$ be the least prime number larger than $\left|V_{i}\right|$. The HH scheme assigns channels to $F_{i}$ by

$f_{i}^{[x, y]}= \begin{cases}v_{i}^{(y)}, & x=0 \text { and } y<\left|V_{i}\right|, \\ \text { an arbitrary element of } V_{i}, & x=0 \text { and } y \geq\left|V_{i}\right|, \\ f_{i}^{[x-1, y]}, & \text { otherwise, }\end{cases}$ where $v_{i}^{(y)}$ is the $y^{t h}$ element in $V_{i}$ (indexed from 0). An example is shown in Fig. 3. Notice that if $\left|V_{i}\right|$ is a prime already, then $\left|F_{i}^{[x]}\right|$ needs to be the next prime number.

The rotating sequence $R_{i}$ is also partitioned into rounds $R_{i}^{[x]}=\left[r_{i}^{[x, 0]}, r_{i}^{[x, 1]}, \cdots, r_{i}^{\left[x,\left|R_{i}^{[x]}\right|-1\right]}\right]$. Let $\left|R_{i}^{[x]}\right|=\left|F_{i}^{[x]}\right|$, the least prime larger than $\left|V_{i}\right|$. The HH scheme assigns channels to $R_{i}$ by

$$
r_{i}^{[x, y]}=f_{i}^{\left[\left(-x \cdot k_{i}+y\right) \bmod \left|R_{i}^{[x]}\right|\right]},
$$

where $k_{i}=\left(\right.$ start $\left._{i} \bmod \left(\left|R_{i}^{[x]}\right|-1\right)\right)+1$. Basically, elements in $R_{i}^{[x]}$ are rotated $k_{i}$ slots forward to produce the next round $R_{i}^{[x+1]}$. An example is shown in Fig. 4. Notice that $r_{i}^{[x, y]}$ and $r_{i}^{[x+1, y]}$ must be different since $1 \leq k_{i} \leq\left|R_{i}^{[x]}\right|-1$. Finally, all slots of the insurance sequence $N_{i}$ are filled in the starting channel start . $_{\text {. }}$

The authors of the HH scheme give the following lemmas:

Lemma 2.3. Let $p$ be a prime and $m$ be an integer coprime with $p$. Then for any $d$, the integers $d, d+m, d+$ $2 m, \cdots, d+(p-1) m$ are all distinct under modulo- $p$ arithmetic. 


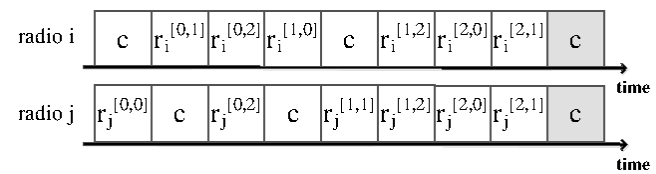

Figure 4: Rounded rotating sequences $R_{i}$ and $R_{j}$ with a common available channel $c$, where $\left|R_{i}^{[x]}\right|=$ $\left|R_{j}^{[x]}\right|=3, k_{i}=1$, and $k_{j}=2$. The MTTR is bounded by $O\left(\left|R_{i}^{[x]}\right|\left|R_{j}^{[x]}\right|\right)$ time slots.

Consider two radios $i$ and $j,\left(V_{i} \backslash P_{i}\right) \cap\left(V_{j} \backslash P_{j}\right) \neq \emptyset$, and two $\mathrm{CH}$ sequences $S_{i}$ and $S_{j}$ adopted by $i$ and $j$ respectively having the same round length.

Lemma 2.4. Given that $S_{i}$ and $S_{j}$ have the fixed sequences $F_{i}$ and $F_{j}$ respectively and slots in $F_{i}$ and $F_{j}$ are timeoverlapping. The MTTR between radios $i$ and $j$ is bounded by $O\left(\left|V_{i}\right|\left|V_{j}\right|\right)$ if $\left|F_{i}^{[x]}\right| \neq\left|F_{j}^{[x]}\right|$.

Lemma 2.5. Given that $S_{i}$ and $S_{j}$ have the rotating sequences $R_{i}$ and $R_{j}$ respectively and slots in $R_{i}$ and $R_{j}$ are time-overlapping. The MTTR between $i$ and $j$ is bounded by $O\left(\left|V_{i}\right|\left|V_{j}\right|\right)$ if $\left|R_{i}^{[x]}\right|=\left|R_{j}^{[x]}\right|$ and $k_{i} \neq k_{j}$.

Based on the above lemmas, the author further show that the $\mathrm{HH}$ scheme guarantees rendezvous for $i$ and $j$ despite of their clock shift, and the MTTR is always bound by $O\left(\left|V_{i}\right|\left|V_{j}\right|\right)$. Interested reader may refer to [19] for the proofs and detailed discussions. It is important to note that Lemmas 2.4 and 2.5 are applicable to $\mathrm{CH}$ sequences generated by any other scheme.

\section{RENDEZVOUS FOR HOMO AND HET- ERO RADIOS}

In this section, we demonstrate the gap between existing homogeneous and heterogeneous $\mathrm{CH}$ schemes and then propose a new rendezvous algorithm, named the Interlocking Channel Hopping (ICH) scheme.

\subsection{The Gap}

Existing homogeneous $\mathrm{CH}$ schemes incur high delay when applied to heterogeneous radios. At the same time, heterogeneous $\mathrm{CH}$ schemes result in severe congestion when applied to homogeneous radios.

To see this, consider the homogeneous $\mathrm{CH}$ schemes first, which assume $V_{i}=V_{j}=V$ and give $O\left(|V|^{2}\right)$ MTTR. When $V_{i} \neq V_{j}$, homogeneous schemes lose guarantee for rendezvous. One extension to these schemes to ensure rendezvous is to let $V_{i}=U$ for all $i$, and those channels in $U \backslash V_{i}$ as $\mathrm{PU}$ occupied (in $P_{i}$ ). However, this leads to $O\left(|U|^{2}\right)$ MTTR, which is unlikely to be acceptable for most of applications as $|U| \gg\left|V_{i}\right|$.

To see the problems of heterogeneous schemes, we need to measure the degree of congestion, called load, incurred by a $\mathrm{CH}$ scheme first.

Definition 3.1 (Channel Load). The channel load of a channel $c$ is defined as $\operatorname{load}_{c h}(c)=\max _{t} \sum_{i \in D} \delta(i, t) /|D|$, where $D$ is the set of all radios and $\delta(i, t)$ is an indicating function that equals to1 if a radio $i$ hops to $c$ at $t$, otherwise 0 .
The $\operatorname{load}_{c h}(c)$ is the proportion of maximum number of radios that hop to $c$ at the same time to the total number of radios, which indicates the degree of congestion of a channel. A CH scheme should result in a low channel load for all c. However, $\operatorname{load}_{c h}(c)$ is dependent with both device capabilities and $\mathrm{CH}$ sequences. To distinguish the load incurred by capabilities and by $\mathrm{CH}$ scheme, we give the following definitions:

Definition 3.2 (Capability Load). The capability load of a set of radios $D$ is defined as load cap $=\max _{c} \sum_{V_{i}} \lambda(i, c) /|D|$, where $V_{i}$ is the capability of radio $i$, and $\lambda(i, c)$ is an indicating function that equals to 1 if $c \in V_{i}$, otherwise 0 .

The $\operatorname{load}_{\text {cap }}$ is the proportion of maximum number of radios capable of sensing the same channel to the total number of radios, which indicates the degree of congestion in the worst. Note that $\operatorname{load}_{c h}(c) \leq \operatorname{load}_{\text {cap }}$ for all $c$, and $\operatorname{load}_{c h}(c *)=\operatorname{load}_{\text {cap }}$ when $c *$ is sensible to the most radios and all these radios hop to $c *$ at the same time.

Definition 3.3 (Load). The load incurred by a $\mathrm{CH}$ scheme is defined as load $=\max _{c} \operatorname{load}_{c h}(c) / \operatorname{load}_{\text {cap }}$.

The load is the proportion of the maximum channel load to the capability load, which measures the degree of congestion incurred by a $\mathrm{CH}$ scheme. Note that a high load does not always imply a high channel load. For example, given a set of heterogeneous radios where the capability-overlaps between these radios are evenly distributed among the radio spectrum, we have a low load cap $_{\text {. Clearly, congestion does }}$ not occur even when load is high, as $\operatorname{load}_{c h}(c) \leq \operatorname{load}_{c a p}$ for all $c$. However, the load is good a measurement of congestion for the networks having high load $_{\text {cap }}$ (e.g., network consisting of homogeneous radios mostly, where load $_{\text {cap }}$ is close to the highest 1 ). In this case, a large number of radios may crowd into a channel, and a high load implies a high channel load (congestion). A CH scheme should keep a low load when load cap is high.

In the following, we focus on homogeneous environments where $\operatorname{load}_{\text {cap }}=1$. Most homogeneous CH schemes proposed recently [21,4] give a balanced load across channels. For example, the JS scheme [14] spreads out the rendezvous opportunities uniformly over the device capability $V$ and time, thus it has an optimal expected load, $E[\text { load }]_{\text {opt }}=$ $E\left[\max _{c} \operatorname{load}_{c h}(c)\right]=E\left[\operatorname{load}_{c h}(c)\right]=\left[\sum_{k=0}^{|D|}\left(\begin{array}{l}|D| \\ k\end{array}\right) \cdot k \cdot\left(\frac{1}{|V|}\right)^{k}(1-\right.$ $\left.\left.\frac{1}{|V|}\right)^{|D|-k}\right] /|D|=\frac{1}{|V|}$, where $k$ is the number of radios hop to channel $c$ in a slot and $\frac{1}{|V|}$ is the probability that a radio hop to channel $c$ in a slot.

When applied to homogeneous environments, some heterogeneous CH schemes [18, 16] lose guarantee for rendezvous. The HH scheme [19], although guaranteeing rendezvous between homogeneous radios, incurs a very high load. This is because that with $\mathrm{HH}$ homogeneous radios rely on their insurance sequences to rendezvous with each other, and the starting channel in $V$ (i.e., start , $_{i}$ which is the same for all $i$ here) is the only channel in these insurance sequences (see Section 2.2). Specifically, the $\mathrm{HH}$ scheme has the expected load $E[\text { load }]_{H H}=E\left[\max _{c} \operatorname{load}(c h(c)]=E\left[\operatorname{load}_{c h}\left(\operatorname{start}_{i}\right)\right]=\right.$ $\frac{2}{3} \cdot \frac{1}{|V|}+\frac{1}{3} \cdot 1=\frac{|V|+2}{3|V|}$, as a) start $i$ is the channel with the highest channel load; b) each $\mathrm{CH}$ sequence has the round length 3 , implying that at a time slot, there are two-third of the radios that hop to channels in the fixed and rotating 
sequences, and one-third of the radios to $s_{a r t}$ in the insurance sequences; c) the fixed and rotating sequences have an optimal load $\frac{1}{|V|}$ as in JS [14], while the in the insurance sequence has the worst load 1 . As we can see, since $\frac{|V|+2}{3|V|}>\frac{1}{3}$, this heterogeneous scheme leads more than $\frac{|D|}{3}$ radios to hop to the starting channel at the same time, results in serious congestion when $|D|$ is large.

It turns out that minimizing the MTTR for heterogeneous radios and minimizing the load for homogeneous radios are two conflicting goals in state of the arts. This severely limits the practicability of $\mathrm{CH}$ schemes, as mixtures of homogeneous and heterogeneous radios are common in real networks.

\subsection{Interlocking Channel Hopping Scheme}

In this subsection, we propose a new scheme, called Interlocking Channel Hopping (ICH) scheme, that minimizes MTTR and congestion for both homogeneous and heterogeneous radios.

The ICH is defined as follows. We partition $S_{i}$ into rounds of length 5 :

$$
s_{i}^{[x, y]}= \begin{cases}f_{i}^{[x]}, & y=0, \\ f_{i}^{[x]}, & y=1, \\ f_{i}^{[x]}, & y=2 \\ r_{i}^{[x]}, & y=3, \\ n_{i}^{[x]}, & y=4,\end{cases}
$$

By repeating the channel in the first three slots, we divide $S_{i}$ into three subsequences, namely the fixed sequence $F_{i}=$ $\left[f_{i}^{[0]}, f_{i}^{[1]}, \cdots\right]$, rotating sequence $R_{i}=\left[r_{i}^{[0]}, r_{i}^{[1]}, \cdots\right]$, and insurance sequence $N_{i}=\left[n_{i}^{[0]}, n_{i}^{[1]}, \cdots\right]$. The $F_{i}$ and $R_{i}$ have the same settings as in the $\mathrm{HH}$ scheme. However, $N_{i}$ is further partitioned into rounds $N_{i}^{[x]}=\left[n_{i}^{[x, 0]}, n_{i}^{[x, 1]}, \cdots\right.$, $\left.n_{i}^{\left[x,\left|N_{i}^{[x]}\right|-1\right]}\right]$. Let $U=\left\{0,1, \ldots,\left|N_{i}^{[x]}\right|-1\right\}$ be the set of slots in a round. We employ the optimal cyclic quorum algorithm [15] to construct a cyclic quorum $Q_{i}, Q_{i} \subseteq U$ and $Q_{i} \neq \emptyset$, for radio $i$.

Definition 3.4 (Coterie). Let $X$ be a set of nonempty subsets of $U$. We call $X$ an coterie iff for all $Q, Q^{\prime} \in X$, $Q \cap Q^{\prime} \neq \emptyset$.

Definition 3.5 (Cyclic Set). Given an integer $l$, where $0 \leq$ $l \leq\left|N_{i}^{[x]}\right|-1$. Let $Q$ be a subset of $U$. We call $C_{l}(Q)$ an $l$-cyclic set of $Q$ iff $C_{l}(Q)=\left\{(q+l) \bmod \left|N_{i}^{[x]}\right|: \forall q \in Q\right\}$.

For convenience, we denote a group of cyclic set as $C(Q)=$ $\left\{C_{l}(Q): \forall l\right\}$.

Definition 3.6 (Cyclic Quorum System). Let $X=\left\{Q_{0}, Q_{1}\right.$, $\cdots\}$ be a set of nonempty subsets of $U$. We call $X$ an cyclic quorum system iff the set of sets $C\left(Q_{0}\right) \cup C\left(Q_{1}\right) \cup \ldots$ is a coterie.

We call elements of $X$ the cyclic quorums. By Definition 3.6 , we have the following theorem.

Theorem 3.7. Given two insurance sequences $N_{i}$ and $N_{j}$, and some cyclic quorums $Q_{j}$ and $Q_{j}$ defined for $N_{i}^{[x]}$ and $N_{j}^{[x]}$ respectively. Despite of clock shift between radios $i$ and $j$, in each round $N_{i}^{[x]}$ (or $N_{j}^{[x]}$ ) there must exists a pair of slots from $Q_{j}$ and $Q_{j}$ that are time-overlapping with each other if $\left|N_{i}^{[x]}\right|=\left|N_{j}^{[x]}\right|$.

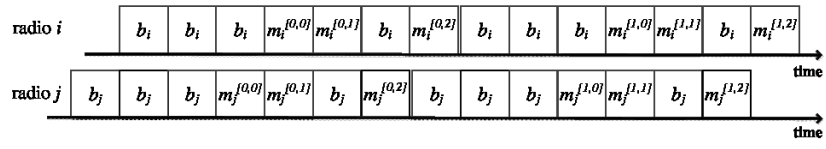

Figure 5: Example insurance sequences $N_{i}$ and $N_{j}$ of the ICH scheme, where $\left|N_{i}^{[x]}\right|=\left|N_{j}^{[x]}\right|=7, Q_{i}=Q_{j}=$ $\{0,1,2,5\}$, and $\left|M_{i}\right|=\left|M_{j}\right|=3$. $B_{i}$ and $B_{j}$ will have time-overlaps every round.

In the ICH scheme, $N_{i}$ is divided into two subsequences, namely sub-rotating sequence $M_{i}$ and sub-insurance sequence $B_{i}$. Let $b_{i}$ be an arbitrary channel in $V_{i}$ chosen by radio $i$, called the insurance channel. We define $N_{i}$ as follows:

$$
N_{i}^{[x, y]}= \begin{cases}m_{i}, & y \notin Q_{i}, \\ b_{i}, & y \in Q_{i},\end{cases}
$$

where $M_{i}=\left[m_{i}^{[0]}, m_{i}^{[1]}, \cdots\right]$, and $B_{i}=\left[b_{i}, b_{i}, \cdots\right]$. The sub-rotating sequence $M_{i}$ is further partitioned into rounds $M_{i}^{[x]}=\left[m_{i}^{[x, 0]}, m_{i}^{[x, 1]}, \cdots, m_{i}^{\left[x,\left|M_{i}^{[x]}\right|\right]}\right]$. Let $\left|M_{i}^{[x]}\right|$ be $\left|N_{i}^{[x]}\right|-$ $\left|Q_{i}\right|$. The ICH scheme assigns channels to $M_{i}$ by

$m_{i}^{[x, y]}= \begin{cases}v_{i}^{(y)}, & x=0 \wedge y<\left|V_{i}\right|, \\ m_{i}^{\left[x-1,\left(y+\left|R_{i}^{[x]}\right|-a_{i}\right) \bmod \left|R_{i}^{[x]}\right|\right],}, & x \neq 0 \wedge y<\left|R_{i}^{[x]}\right|, \\ v_{i}^{\left(x \bmod \left|V_{i}\right|\right),} & \text { otherwise, }\end{cases}$

where $v_{i}^{(y)}$ is the $y^{t h}$ element in $V_{i}$ (indexed from 0 ) and $a_{i}$ is the rotating amount of $M_{i}^{[x]}$. Basically, we fill $B_{i}$ with $b_{i}$, and $M_{i}^{[x]}$ is similar to the rotating sequence $R_{i}^{[x]}$, except that the rotating amount $a_{i}$ of is determined by the insurance channel $b_{i}$, i.e., $a_{i}=b_{i}\left(\bmod \left(\left|M_{i}^{[x]}\right|-1\right)\right)+1$. An example is shown in Fig. 5. We let $\left|N_{i}^{[x]}\right|$ be a prime number such that $\left|N_{i}^{[x]}\right|-\left|Q_{i}\right| \geq\left|R_{i}^{[x]}\right|$, and employ the optimal cyclic quorum algorithm [15] to construct $N_{i}^{[x]}$, that we put $B_{i}$ in the quorum positions $Q_{i}$. Due to the space limitation, we do not discuss the construction of cyclic quorum systems here. Interested reader may refer to [15].

Theorem 3.7 guarantees that there are time-overlapping slots between $B_{i}$ and $B_{j}$. With insurance sequences $N_{i}$ and $N_{j}$, two radios will rendezvous at either the time-overlaping slot pair (denoted by $\left(B_{i}, B_{j}\right)$ ) if they choose the same insurance channel, or otherwise at the $\left(M_{i}, M_{j}\right),\left(M_{i}, B_{j}\right)$, or $\left(B_{i}, M_{j}\right)$ pairs using Lemma 2.5.

However, the problem is how to choose the insurance channel $b_{i}$. Considering two radios $i$ and $j$ with $\left|F_{i}^{[x]}\right|=\left|F_{j}^{[x]}\right|$ and start $_{i}=$ start $_{j}$, as $V_{i}$ and $V_{j}$ may still be different, $V_{i}$ needs to pick $b_{i}$ such that $b_{i} \in V_{i} \cap V_{j}$ without knowing $V_{j}$ in advance, and vise versa for $V_{j}$ to pick $s_{j}$. We let $p=\left|F_{i}^{[x]}\right|=\left|F_{j}^{[x]}\right|$ and $q_{i}\left(q_{j}\right)$ be the largest prime number that smaller than $\left|V_{i}\right|\left(\left|V_{j}\right|\right)$. As we know that $\left|F_{i}^{[x]}\right|$ is the least prime number that larger than $\left|V_{i}\right|$, we have $q_{i}=q_{j}=q$ and $q<\left|V_{i}\right|,\left|V_{j}\right|<p$ given $\left|F_{i}^{[x]}\right|=\left|F_{j}^{[x]}\right|$. Hence, $V_{i}$ can be sure that $\left[\right.$ start $_{i}$, start $\left._{i}+q_{i}-1\right] \subseteq V_{i} \cap V_{j}$ and can pick any element in $\left[\right.$ start $_{i}$, start $\left._{i}+q_{i}-1\right]$ as $b_{i}$. Similarly $V_{j}$ can pick $b_{j}$ from $\left[\right.$ start $_{j}$, start $\left._{j}+q_{j}-1\right]$.

Finally, the ICH scheme assigns the insurance channel to the sub-insurance sequence. Note that, $b_{i}$ cannot be selected from occupied channels by PUs, i.e., $b_{i} \in\left(V_{i} \backslash P_{i}\right)$. Accord- 


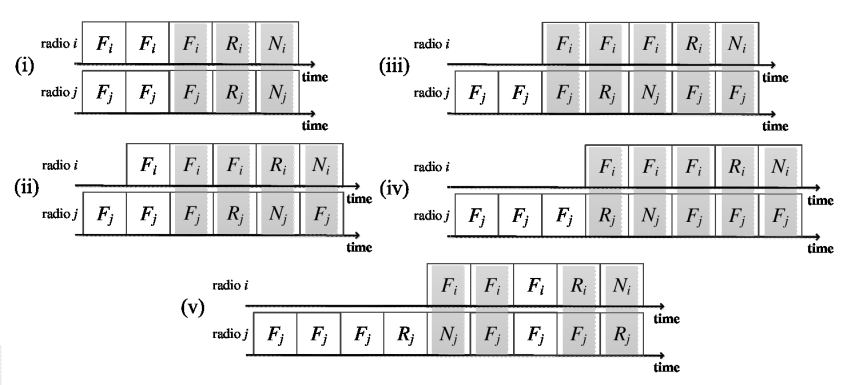

Figure 6: Each round of a $\mathbf{C H}$ sequence $S_{i}$ of the ICH scheme is partitioned into three consecutive $F_{i}$ s followed by a $R_{i}$ and a $N_{i}$. In terms of time-overlapping slots, the shifts between two $\mathrm{CH}$ sequences range from 0 to 4 slots.

ingly, we allow radios to use different insurance channels even when $s_{a r t}=$ start $_{j}$, thereby avoiding homogeneous radios crowding into the the same channel.

\subsection{Minimizing Delay}

Next, we verify that the MTTR of ICH is $O\left(\left|V_{i}\right|\left|V_{j}\right|\right)$ for heterogeneous radios and $O\left(|V|^{2}\right)$ for homogeneous radios. Firstly, let's discuss the MTTR using the time-overlapping slots between $N_{i}$ and $N_{j}$. Considering two radios $i$ and $j$ with $\left|F_{i}^{[x]}\right|=\left|F_{j}^{[x]}\right|$ and start $_{i}=$ start $_{j}$, we discuss two cases: 1) $\left.b_{i}=b_{j}, 2\right) b_{i} \neq b_{j}$.

- Case 1: By Theorem 3.7, when $\left|N^{[x]}\right|=\left|N_{i}^{[x]}\right|=$ $\left|N_{j}^{[x]}\right|$, there is at least one time-overlapping in a round $\left(\left|N^{[x]}\right|\right.$ slots) between $B_{i}$ and $B_{j}$, and $i$ and $j$ rendezvous as $b_{i}=b_{j}$. By [15], we have $\left|N_{i}^{[x]}\right| \leq 2\left|R_{i}^{[x]}\right|=$ $O\left(\left|V_{i}\right|\right)$, that is, the MTTR of this case is $\bar{O}\left(\left|V_{i}\right|\right)$.

- Case 2: $b_{i} \neq b_{j}$ implies $a_{i} \neq a_{j}$. If $M_{i}$ time-overlaps $M_{j}$ entirely, by Lemma 2.5 , the MTTR is $O\left(\left|M_{i}^{[x]}\right|\right.$ $\left.\left|M_{j}^{[x]}\right|\right)=O\left(\left|V_{i}\right|\left|V_{j}\right|\right)$. Otherwise, considering the timeoverlapping pair $\left(M_{i}, B_{j}\right)$, by Lemmas 2.3 and $2.5, b_{j}$ will meet all different channels in $M_{i}$ and $b_{j} \in V_{i}$, thus $i$ and $j$ will rendezvous in $O\left(\left|M_{i}^{[x]}\right|\left|N_{j}^{[x]}\right|\right)=O\left(\left|V_{i}\right|\left|V_{j}\right|\right)$ slots, vise versa for time-overlapping pair $\left(B_{i}, M_{j}\right)$.

Theorem 3.8. Given that two radios $i$ and $j$ with common available channels that adopt two $C H$ sequences generated by the ICH scheme with $N_{i}, N_{j}$ time-overlapping. The MTTR between $i$ and $j$ is bounded by $O\left(\left|V_{i}\right|\left|V_{j}\right|\right)$ as long as a) $\left(V_{i} \backslash P_{i}\right) \cap\left(V_{j} \backslash P_{j}\right) \neq \emptyset$ and b) $\left|F_{i}^{[x]}\right|=\left|F_{j}^{[x]}\right|$ and start $_{i}=$ start $_{j}$.

Then, as shown in Fig. 6, we verify the MTTR of this ICH scheme by considering three time-overlapping cases: (i) $F_{i}$ with $F_{j}, R_{i}$ with $R_{j}$, and $N_{i}$ with $N_{j}$, (ii) $F_{i}$ with $F_{j}, F_{i}$ with $R_{j}, R_{i}$ with $N_{j}$, and $N_{i}$ with $F_{j}$, (iii) $F_{i}$ with $F_{j}, F_{i}$ with $R_{j}, F_{i}$ with $N_{j}, R_{i}$ with $F_{j}$ and $N_{i}$ with $F_{j}$. Notice that the case (iv) in Fig. 6 is covered by case (iii), and case (v) is covered by the (ii). Without loss of generality, each of the above cases can be further classified into three subcases in terms of capabilities of radios $i$ and $j$ : (a) $\left|V_{i}\right| \neq\left|V_{j}\right|$, (b) $\left|V_{i}\right|=\left|V_{j}\right| \wedge$ start $_{i} \neq$ start $_{j}$, and (c) $\left|V_{i}\right|=\left|V_{j}\right| \wedge$ start $_{i}=$ start $_{j}$. Since $\left|F_{i}^{[x]}\right|$ depends on $\left|V_{i}\right|$, we rewrite the cases as: (a) $\left|F_{i}^{[x]}\right| \neq\left|F_{j}^{[x]}\right|$, (b) $\left|F_{i}^{[x]}\right|=\left|F_{j}^{[x]}\right| \wedge$ start $_{i} \neq$ start $_{j}$ (c) $\left|F_{i}^{[x]}\right|=\left|F_{j}^{[x]}\right| \wedge$ start $_{i}=$ start $_{j}$. We assume $\left(V_{i} \backslash P_{i}\right) \cap$ $\left(V_{j} \backslash P_{j}\right) \neq \emptyset$ and verify the MTTR of the ICH scheme for each of these subcases.

- Case (i-a) \& (ii-a) \& (iii-a): Since $F_{i}$ time-overlaps $F_{j}$ and $\left|F_{i}^{[x]}\right| \neq\left|F_{j}^{[x]}\right|$, by Lemma 2.4, we have $O\left(\left|V_{i}\right|\left|V_{j}\right|\right)$ MTTR.

- Case (i-b): Since $R_{i}$ time-overlaps $R_{j}$ and $\left|R_{i}^{[x]}\right|=$ $\left|R_{j}^{[x]}\right| \wedge$ start $_{i} \neq$ start $_{j}$, which implies $k_{i} \neq k_{j}$, by Lemma 2.5, we have $O\left(\left|V_{i}\right|\left|V_{j}\right|\right)$ MTTR.

- Case (i-c): Since $N_{i}$ time-overlaps $N_{j}$ and $\left|F_{i}^{[x]}\right|=$ $\left|F_{j}^{[x]}\right| \wedge$ start $_{i}=$ start $_{j}$, by Theorem 3.8, we have $O\left(\left|V_{i} \| V_{j}\right|\right)$ MTTR.

- Case (ii-b) \& (iii-b): Since $F_{i}$ time-overlaps $R_{j}$ and $\left|F_{i}^{[x]}\right|=\left|R_{j}^{[x]}\right| \wedge$ start $_{i} \neq$ start $_{j}$, which implies $k_{i}^{\prime} \neq k_{j}$ by viewing the rotating amount of $F_{i}$ as $k_{i}^{\prime}=0$ and $k_{j} \in\left[1,\left|R_{j}^{[x]}\right|-1\right]$, by Lemma 2.5 , we have $O\left(\left|V_{i}\right|\left|V_{j}\right|\right)$ MTTR.

- Case (ii-c) \& case (iii-c): $N_{i}$ time-overlaps $F_{j}$ and $\left|F_{i}^{[x]}\right|=\left|F_{j}^{[x]}\right| \wedge$ start $_{i}=$ start $_{j}$, which implies $\left|N_{i}^{[x]}\right|$, $\left|F_{j}^{[x]}\right|$ coprime and $s_{i} \in V_{j}$ (i.e., $\left\{s_{i}\right\} \cap V_{j} \neq \emptyset$ ). Also, the positions of $s_{i}$ are fixed in $N_{i}^{[x]}$, by Lemma 2.4, we have $O\left(\left|V_{i}\right|\left|V_{j}\right|\right)$ MTTR.

In summary of all the above cases, the MTTR of ICH is bounded by $O\left(\left|V_{i}\right|\left|V_{j}\right|\right)$ for heterogeneous radios $\left(V_{i} \neq V_{j}\right)$ and $O\left(|V|^{2}\right)$ for homogeneous radios $\left(V_{i}=V_{j}=V\right)$. Note that, let $V_{i}^{\prime}=V_{i} \backslash P_{i}$, the $\mathrm{CH}$ sequence, which is constructed on $V_{i}^{\prime}$ by setting start $_{i}$ and last $_{i}$ be the first and last unoccupied channels respectively and $\left|V_{i}^{\prime}\right|=$ last $_{i}-$ start $_{i}+1$, still has the same rendezvous guarantee with shorter TTR, due to $\left|V_{i}^{\prime}\right| \leq\left|V_{i}\right|$.

\subsection{Minimizing Congestion}

To demonstrate ICH minimizes the congestion, we conduct a series of simulations and compare ICH with $\mathrm{HH}$ and JS in terms of the degree of congestion, namely load. First, we show that the load is minimized for mixtures of heterogeneous radios in Fig. 14(b), where ICH has the load as low as the JS's load, which is the optimal load. Also we examine the worst case for load. Fig. 16 (b) exhibits the load induced by a set of homogeneous radios versus the number of radios. ICH has $25 \%$ to $40 \%$ reduction from $\mathrm{HH}$ in load as increasing the number of radios, and we can expect that the reduction will be higher when there are more radios. Stand by the simulation results, ICH is shown to minimize the congestion and have the load close to the optimal load.

\section{EXPERIMENTAL RESULTS}

In this section, we evaluate the performance of Interlocking Channel Hopping ( $\mathrm{ICH})$ scheme. We compare ICH with the state of the arts in homogeneous and heterogeneous environments, respectively Jump-Stay (JS) [14] and the $\mathrm{HH}$ scheme [19]. According to the assumption mentioned in Sec. 3.1 that $V_{i}=V_{j}=U$, we let JS generates $\mathrm{CH}$ sequences based on $U$. Note that we do not compare our study with other works due to loss of guarantee and their limitations stated in Sec. 5. We investigate the basic behaviors and proper functioning of compared schemes by pairwise radios, 
and study the performance for mixtures of homogeneous and heterogeneous radios with TTR and load. We also verify the reduction on load compared with the HH scheme in the homogeneous environment.

Since timer synchronization is hard to achieve in practice, we focus on the asynchronous environment. We implement these works based on the Network Simulator 3 (NS3). WiFi $\mathrm{MAC}$ is modified to support the channel hopping function, and the IEEE $802.11 \mathrm{~b}$ is adopted as the MAC layer protocol. Each radio is capable to switch amongst channels in its device capability, and is either receiving or transmitting at a time. Also, radios can detect whether a channel is idle or not. Considering the large range of currently available spectrum which is up to $3 \mathrm{GHz}$ or higher, we assume $5 \mathrm{MHz}$ per channel and set the number of universal channels $|U|$ to 600 by default. The period of a time slot is set to $10 \mathrm{~ms}$. The ratio of non-idle channels to the universal channels is set to 0.1 , where those non-idle channels are randomly selected from $U$. We set the default average capability $|V|=25$, and control the overlapping ratio, which is the proportion of channels that can be operated by more than two radios to the union of capability $\bigcup_{i} V_{i}$. We set the overlapping ratio to 0.1. We focus on the affects of capabilities of radios, to avoid unnecessary complexity, we let each radio's transmission range cover other radios. Note that, a possible rendezvous is treated as a failed rendezvous if it spends more than 600 seconds, and it is not counted into average TTR.

\subsection{Pairwise Radios}

We verify the rendezvous guarantee of ICH and observe its fundamental behaviors in this series of simulation. Two radios $i$ and $j$ are used in a simulation run and each data point is averaged from 120 runs. We generate two different range of capabilities that $|V|+x,|V|-x$, where $x$ is randomly selected from $[1,|V| / 2]$. First, we vary the overlapping ratio from 0.5 to 0.1 . As the lower overlapping ratio indicating less common operable channels, intuitively, the TTR may get larger. In Fig. 7(a), the average TTR of JS substantially increases from 10 to 17 seconds, on the contrary, the average TTR of ICH and HH have no obvious growth. We can see the advantage that construct $\mathrm{CH}$ sequence by capability instead of universal channels. Fig. 7(b) shows that all these schemes can guarantee rendezvous. Since JS construct CH sequences based on $U$, it incurs large TTR but does not lose guarantee. Fig. 8 shows that how many ratio of rendezvous can be achieved in 60 seconds. While overlapping ratio is 0.1 , JS has most TTRs larger than ICH's. Moreover, JS has more excessively large TTRs, showing that the infeasibility and instability from $U$ affects the average TTR greatly. The $\mathrm{HH}$ scheme has much more rendezvous than others in the first five second. One of reasons is that, while overlapping ratio is not high, the insurance sequence containing only one channel has much higher probability to rendezvous. This advantage of the $\mathrm{HH}$ scheme becomes not obvious while overlapping ratio is 0.5 .

Then, we change non-idle channel ratio from 0.1 to 0.5 . The results are shown in Fig. 9. The effect of non-idle channel ratio is similar to overlapping ratio, that the number of common operable channels decreases while non-overlapping ratio increases. As we can see, the trends of average TTR and success rate are similar to the ones in varying overlapping ratio, and we believe that the reasons are the same. Although the one-channel insurance sequence of the $\mathrm{HH}$

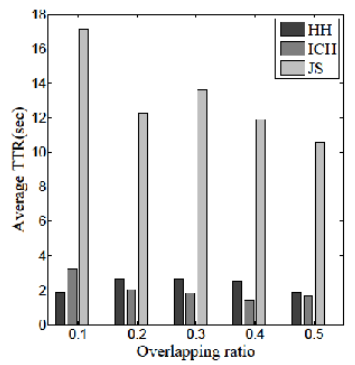

(a)

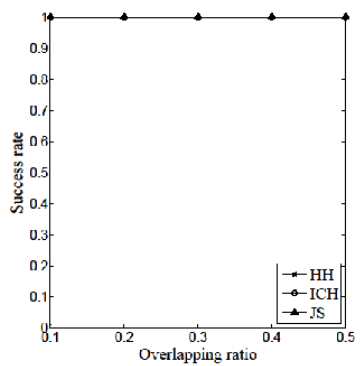

(b)
Figure 7: (a) average TTR vs. overlapping ratio (b) success rate vs. overlapping ratio

(a)

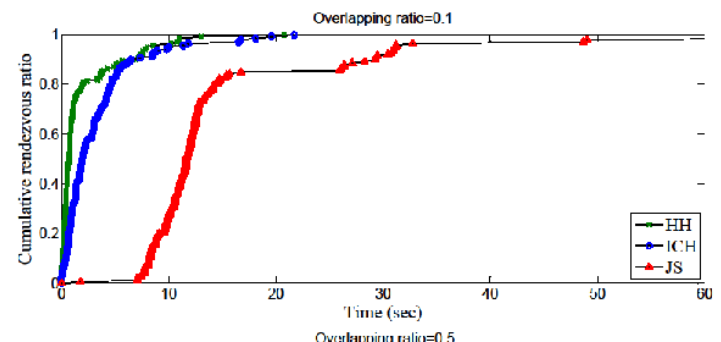

(b)

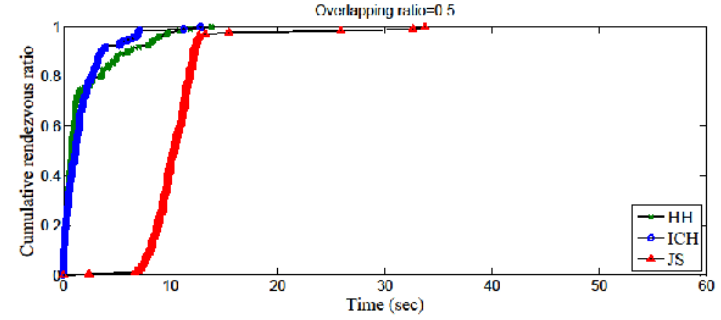

Figure 8: Cumulative rendezvous ratio vs. time while (a) overlapping ratio $=0.1$ and (b) 0.5 respectively

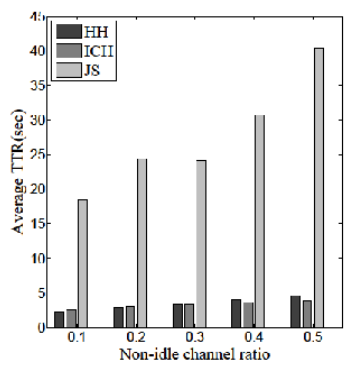

(a)

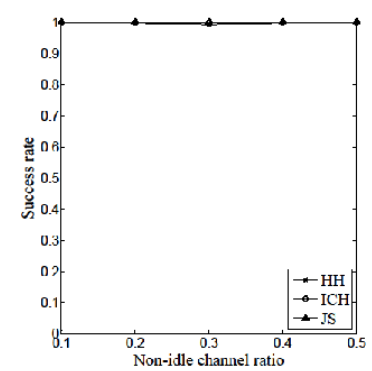

(b)
Figure 9: (a) average TTR vs. non-idle channel ratio (b) success rate vs. non-idle channel ratio 


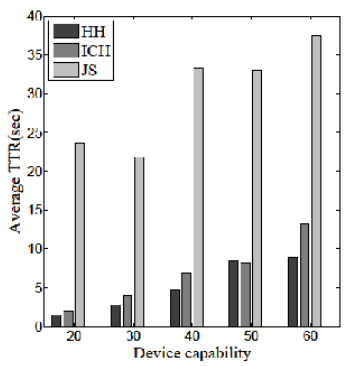

(a)

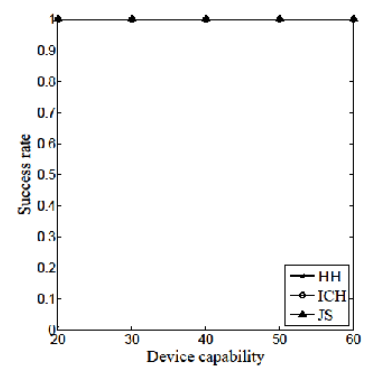

(b)
Figure 10: (a) average TTR vs. device capability (b) success rate vs. device capability (a)

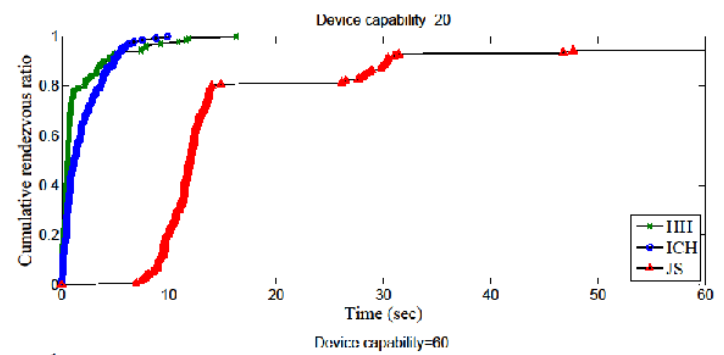

(b)

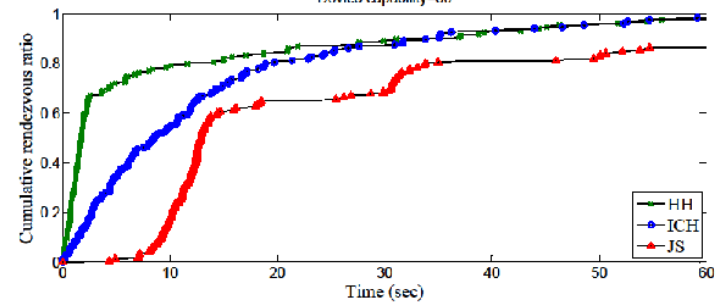

Figure 11: Cumulative rendezvous ratio vs. time while (a) device capability $=20$ and (b) 60 respectively

scheme may speed up the TTR, it takes a risk that onethird of the time will be wasted while the starting channel is non-idle. In the Fig. 9(b), we find that the success rate of the $\mathrm{HH}$ is not 1 while non-idle ratio is 0.3 . We believe that is because non-idle insurance channel delays TTR to be larger than 600 seconds. Another reason may be most of common operable channels happen to be non-idle in that case.

Next, we change average capability $|V|$ from 20 to 60 . We may think that the performance of JS may be better while $|V|$ is closer to $|U|$, where the environment seems more homogeneous. The results are shown in Fig. 10. The TTR of ICH and $\mathrm{HH}$ increase as we expected, but TTR of JS also increases due to the heterogeneity of radios. However, ICH and HH still have much lower TTR. Again, the advantage of one-channel insurance sequence of the $\mathrm{HH}$ scheme appears in Fig. 11, that reduces much of its average TTR. Overall, the ICH scheme have better performance while $V_{i} \neq V_{j}$.

\subsection{Mixtures of Homogeneous and Heteroge- neous Radios}

In this set of simulation, we generate three kinds of heterogeneous radios and five radios for each kind. Similar to the trends in the above pairwise cases, in Fig. 12(a) and

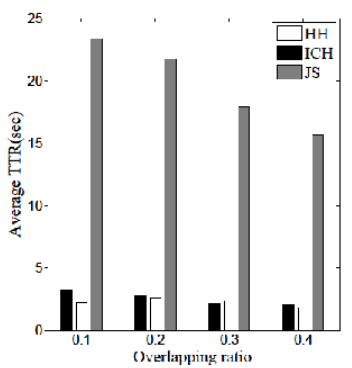

(a)

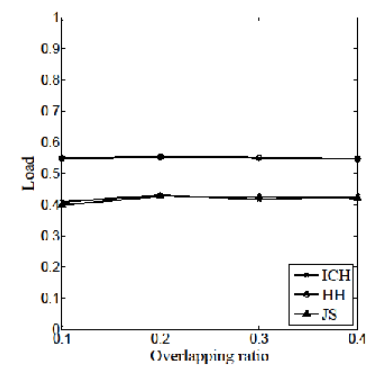

(b)
Figure 12: Mixtures of homo \& hetero radios: (a) average TTR vs. overlapping ratio (b) load vs. overlapping ratio

(a)

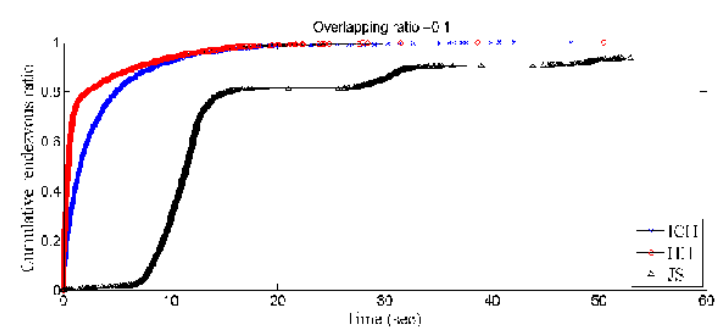

(b)

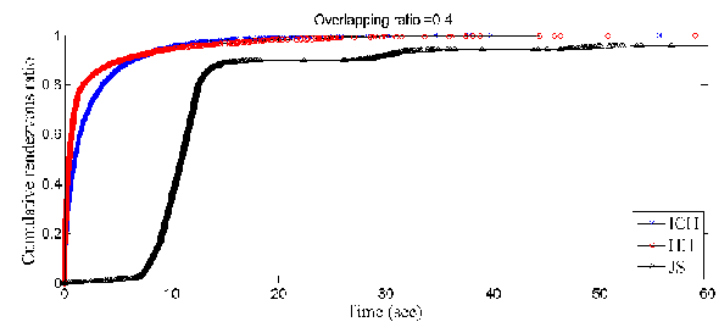

Figure 13: Mixtures of homo \& hetero radios: cumulative rendezvous ratio vs. time while (a) overlapping ratio $=0.1$ and (b) 0.4 respectively

14(a), TTR of ICH and the HH are stable to overlapping ratio and non-idle ratio while TTR of JS changes largely. Shown in Fig. 13 and 15, ICH can complete all possible rendezvous within one minute while JS cannot. ICH even can achieve 90\% rendezvous in 10 seconds. In Fig. 12(b) and 14 (b), we show that ICH reduces the load by $25 \%$ from $\mathrm{HH}$ for heterogeneous radios, and the load of ICH is low as JS. Note that, mentioned in Sec. 3.1, JS is load-balanced and has optimal load. On the whole, ICH has low TTR and low load for mixtures of heterogeneous and homogeneous radios.

\subsubsection{Worst Case for Load}

As mentioned in Sec. 3.1, the load will be worst while the mixtures consisting of homogeneous radios only. We verify the load reduction of $\mathrm{ICH}$ compared to the $\mathrm{HH}$ in the homogeneous environment. We vary the number of homogeneous radios from 10 to 40, as exhibited in Fig. 16, while the average TTR of ICH is competitive with $\mathrm{HH}$, the ICH largely reduces the load by $35 \%$ to $50 \%$ from the $\mathrm{HH}$. This is because that the congestion gets severer quickly due to the high load of the $\mathrm{HH}$ scheme. The load non-intuitively decreases while number of radios increases, however, the number of radios, 


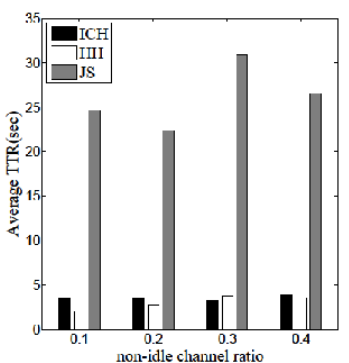

(a)

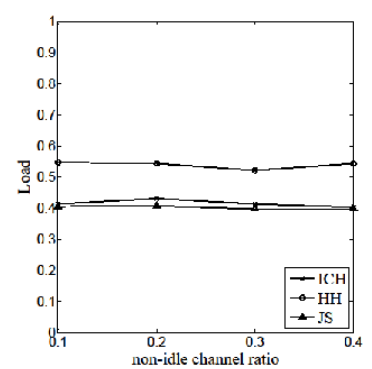

(b)
Figure 14: Mixtures of homo \& hetero radios: (a) average TTR vs. non-idle channel ratio (b) load vs. non-idle channel ratio

(a)

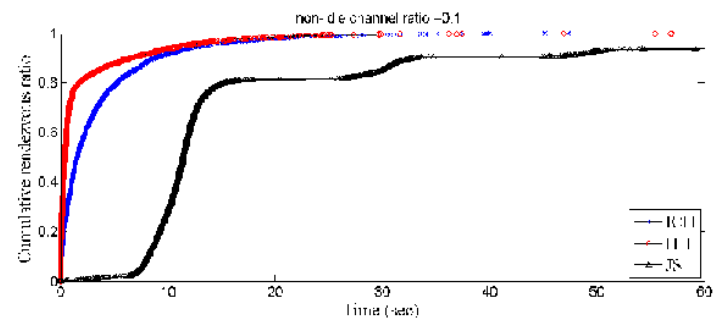

(b)

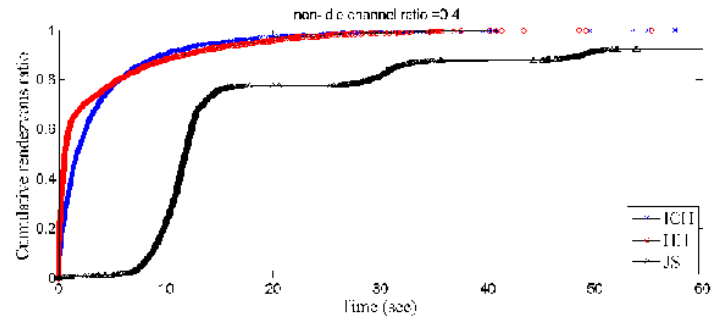

Figure 15: Mixtures of homo \& hetero radios: cumulative rendezvous ratio vs. time while (a) non-idle channel ratio $=0.1$ and $(\mathrm{b}) 0.4$ respectively

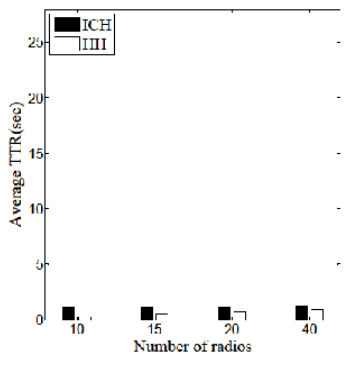

(a)

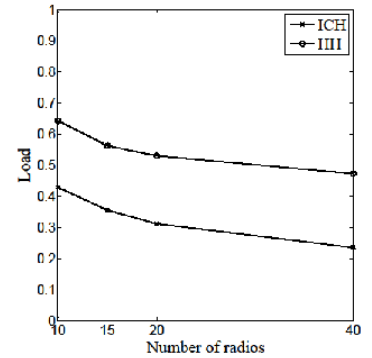

(b)
Figure 16: Multiple homogeneous radios: (a) average TTR vs. number of radios (b) load vs. number of radios

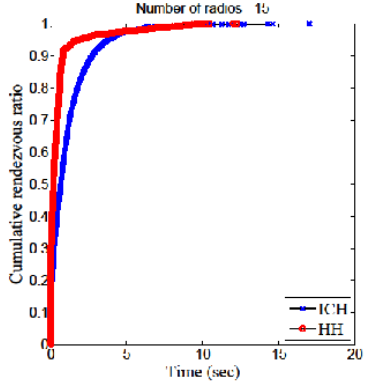

(a)

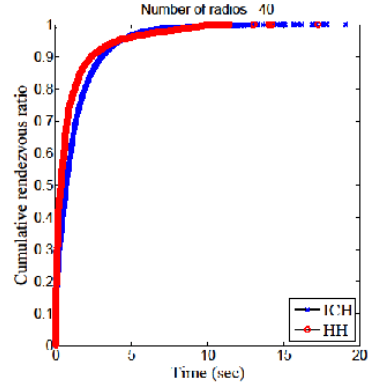

(b)
Figure 17: Multiple homogeneous radios: cumulative rendezvous ratio vs. time while number of radios $=15$ and 40 respectively

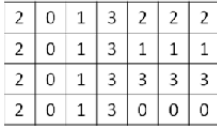

(a)

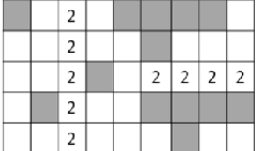

(b)
Figure 18: (a) The mapping grid of $V_{i}=\{0,1,2,3\}$ (b) Let $\left|V_{j}\right|=5$ and $V_{i} \cap V_{j}=\{2\}$, the mapping grid of $V_{j}$ only shows assignments of channel 2 , and marks the corresponding slots $V_{i}$ hops to channel 2. It cannot guarantee rendezvous in $O\left(\left|V_{j}\right|^{2}\right)$ (or $\left.O\left(\left|V_{i}\right|\left|V_{j}\right|\right)\right)$ slots.

which hop to the same channel at the same slot, increases. Therefore the congestion becomes more serious when using the $\mathrm{HH}$ scheme. Also, the $\mathrm{HH}$ has impact of congestion on its immediate slowing down of TTR, where the up-left angle becomes smooth as seen in Fig. 17. This set of simulations show that the $\mathrm{ICH}$ is robust to congestion.

\section{RELATED WORK}

The typical blind rendezvous technique is Channel Hopping $(\mathrm{CH})$. Traditionally, $\mathrm{CH}$ schemes for homogeneous radios can be divided into synchronous and asynchronous depend on their environments. In the synchronous environment, the timer is synchronized among all the radios, so that all the radios can start and hop to a channel simultaneously $[13,20,17,3]$. However, timer synchronization may not be easily achieved in practice. Asynchronous homogeneous $\mathrm{CH}$ schemes are proposed. Bian et al. [5, 4] proposed A-MOCH, and $\mathrm{Sym}-\mathrm{ACH}$. A-MOCH requires senders and receivers are known in advance which are not likely known before rendezvous. Sym-ACH assumes each node has an unique ID, and the MAC address seems the only choice, causing the $2^{48} \mathrm{O}\left(|V|^{2}\right)$ MTTR. DaSilva et al. [10] proposed another $\mathrm{CH}$ scheme under the condition that all radios have the same available channels. Zhang et al. [21] proposed the AsynETCH that needs to pre-construct possible schedules with guaranteed rendezvous, but it is not likely to pre-construct overlapping schedules while $V_{i} \neq V_{j}$ and $V_{j}$ is unknown by $i$. Lin et al. [14] proposed the JS that uses jump and stay patterns, with length of $2|V|$ and $|V|$ respectively, to ensure rendezvous even when timers are not synchronized. 
For asynchronous heterogeneous environments, Theis et al. proposed the MC [18] based on the number theory. The MC scheme and its modified version cannot guarantee rendezvous if two radios accidentally make the same decision to construct their $\mathrm{CH}$ sequences either on the "rate" parameter or on the augmented capability. Romaszko et al. proposed the MtQS-DSrdv [16] that verifies the rendezvous when $\left|V_{i}\right|,\left|V_{j}\right| \leq 8$, but does not provide a theoretical guarantee for all cases under heterogeneous environments. Since the search method for difference set is not detailed in [16], we simplify the MtQS-DSrdv as follows: each radios $i$ i) creates a slot to channel mapping grid of size $\left|V_{i}\right| \cdot\left(2\left|V_{i}\right|-1\right)$, where each row $r$ has slots from $t_{i}^{\left[0+r\left(2\left|V_{i}\right|-1\right)\right]}$ to $t_{i}^{\left[2\left|V_{i}\right|-2+r\left(2\left|V_{i}\right|-1\right)\right]}$, ii) assigns each channel in $V_{i}$ to a randomly selected column of slots from the first $\left|V_{i}\right|$ columns, iii) assigns each channel in $V_{i}$ to a randomly selected rows of the rest empty slots. An example is depicted in the Fig. 18 (a). While $V_{i} \neq V_{j}$, the mapping grids need to be pre-constructed to ensure rendezvous, however, it is not sure to find the mapping grids with guarantee. We show a failed example in the Fig. 18 (b).

\section{CONCLUSION}

In this paper, we study the channel hopping schemes for homogeneous and heterogeneous cognitive radios. We proposed the ICH scheme, an efficient channel hopping scheme which minimizes MTTR and congestion for homogeneous and heterogeneous radios. Extensive simulations showed that our proposal achieves 10 times faster TTR than the state-of-the-art homogeneous scheme and $50 \%$ reduction of congestion from the state-of-the-art heterogeneous scheme.

\section{REFERENCES}

[1] I.F. Akyildiz, W.Y. Lee, M.C. Vuran, and S. Mohanty. Next generation/dynamic spectrum access/cognitive radio wireless networks: a survey. Computer Networks, 50(13):2127-2159, 2006.

[2] R. Bagheri, A. Mirzaei, S. Chehrazi, M. Heidari, M. Lee, M. Mikhemar, M. Tang, and A. Abidi. An $800 \mathrm{mhz}$ to $5 \mathrm{ghz}$ software-defined radio receiver in 90nm cmos. In IEEE Int'l Solid-State Circuits Conference (ISSCC), pages 1932-1941, 2006.

[3] P. Bahl, R. Chandra, and J. Dunagan. Ssch: slotted seeded channel hopping for capacity improvement in ieee 802.11 ad-hoc wireless networks. In Proc. of $A C M$ Int'l Conf. on Mobile computing and networking (MobiCom), pages 216-230, 2004.

[4] K. Bian and J. Park. Maximizing rendezvous diversity in rendezvous protocols for decentralized cognitive radio networks. IEEE Transactions on Mobile Computing, 2012.

[5] K. Bian, J.M. Park, and R. Chen. Control channel establishment in cognitive radio networks using channel hopping. IEEE Journal on Selected Areas in Communications, 29(4):689-703, 2011.

[6] V. Brik, E. Rozner, S. Banerjee, and P. Bahl. Dsap: a protocol for coordinated spectrum access. In IEEE Int'l Symp. on New Frontiers in Dynamic Spectrum Access Networks (DySPAN), pages 611-614, 2005.

[7] M.M. Buddhikot, P. Kolodzy, S. Miller, K. Ryan, and J. Evans. Dimsumnet: new directions in wireless networking using coordinated dynamic spectrum. In
IEEE Int'l Symp. on World of Wireless Mobile and Multimedia Networks (WoWMoM), pages 78-85, 2005.

[8] D. Cabric, S.M. Mishra, and R.W. Brodersen. Implementation issues in spectrum sensing for cognitive radios. In Proc. of Asilomar Conf. on Signals Systems and Computers, pages 772-776, 2004.

[9] C. Cormio and K.R. Chowdhury. Common control channel design for cognitive radio wireless ad hoc networks using adaptive frequency hopping. Ad Hoc Networks, 8(4):430-438, 2010.

[10] L.A. DaSilva and I. Guerreiro. Sequence-based rendezvous for dynamic spectrum access. In IEEE Int'l Symp. on New Frontiers in Dynamic Spectrum Access Networks (DySPAN), pages 1-7, 2008.

[11] A. Ghasemi and E.S. Sousa. Spectrum sensing in cognitive radio networks: requirements, challenges and design trade-offs. IEEE Communications Magazine, 46(4):32-39, 2008.

[12] M. Ingels, V. Giannini, J. Borremans, G. Mandal, B. Debaillie, P. Van Wesemael, T. Sano, T. Yamamoto, D. Hauspie, J. Van Driessche, et al. A $5 \mathrm{~mm}^{2} 40 \mathrm{~nm}$ lp cmos transceiver for a software-defined radio platform. IEEE Journal of Solid-State Circuits, 45(12):2794-2806, 2010.

[13] Y.R. Kondareddy and P. Agrawal. Synchronized mac protocol for multi-hop cognitive radio networks. In Proc. of IEEE Int'l Conf. on Communications (ICC), pages 3198-3202, 2008.

[14] Z. Lin, H. Liu, X. Chu, and Y.W. Leung. Jump-stay based channel-hopping algorithm with guaranteed rendezvous for cognitive radio networks. In Proc. of IEEE Int'l Conf. on Computer Communications (INFOCOM), pages 2444-2452, 2011.

[15] W.S. Luk and T.T. Wong. Two new quorum based algorithms for distributed mutual exclusion. In Proc. of IEEE Int'l Conf. on Distributed Computing Systems (ICDCS), pages 100-106, 1997.

[16] S. Romaszko and P. Mahonen. A rendezvous protocol with the heterogeneous spectrum availability analysis for cognitive radio ad hoc networks. Journal of Electrical and Computer Engineering, 2012.

[17] C.F. Shih, T.Y. Wu, and W. Liao. Dh-mac: A dynamic channel hopping mac protocol for cognitive radio networks. In Proc. of IEEE Int'l Conf. on Communications (ICC), pages 1-5, 2010.

[18] N.C. Theis, R.W. Thomas, and L.A. DaSilva. Rendezvous for cognitive radios. IEEE Transactions on Mobile Computing, 10(2):216-227, 2011.

[19] S.H. Wu, C.C. Wu, and W.K. Han. Rendezvous for heterogeneous cognitive radios. In http://www.cs.nthu.edu.tw/ shwu/reports/shwu-tr12hh.pdf.

[20] C. Xin, M. Song, L. Ma, and C.C. Shen. An approximately optimal rendezvous scheme for dynamic spectrum access networks. In Proc. of IEEE Int'l Conf. on Gloal Telecommunications (GLOBECOM), pages 1-5, 2011.

[21] Y. Zhang, Q. Li, G. Yu, and B. Wang. Etch: Efficient channel hopping for communication rendezvous in dynamic spectrum access networks. In Proc. of IEEE Int'l Conf. on Computer Communications (INFOCOM), pages 2471-2479, 2011. 